Leonardo Domingos Biagio

(- https://orcid org/0000-0002-7494-1394

Priscila Moreira²

Ohttps://orcid.org/0000-0002-5898-8512

Cristiane Kovacs Amaral ${ }^{1}$

- https://orcid.org/0000-0002-3575-0800

\section{Comportamento alimentar em obesos e sua correlação com o tratamento nutricional}

\author{
Eating behavior in obesity and its correlation with nutritional treatment
}

DOl: $10.1590 / 0047-2085000000280$

\section{RESUMO}

Objetivo: Avaliar os tipos de comportamentos alimentares em indivíduos com obesidade e correlacionar com a adesão ao tratamento proposto. Métodos: Estudo transversal desenvolvido em instituição de cardiologia em indivíduos com obesidade. Os tipos de comportamentos alimentares foram analisados pela escala The Three Factor Eating Questionnaire - R21 (TFEQ-21) - versão traduzida e adaptada para brasileiros. Nela são abordadas três subescalas: restrição cognitiva (RC), alimentação emocional (AE) e descontrole alimentar (DA). A adesão ao tratamento nutricional foi verificada pelo instrumento desenvolvido pela instituição, baseado nas principais diretrizes de doenças crônicas. Resultados: Analisaram-se 100 indivíduos, com maior prevalência do sexo feminino (68\%). Em relação à adesão, somente $25 \%$ apresentam boa aderência (escore > 60\%). Foi possível identificar a relação entre o IMC e a RC; quanto maior o IMC, menor foi a intensidade da RC $(p=0,02)$. Observou-se correlação positiva entre a RC e adesão ao consumo de gorduras $(p=0,02)$ e fibra alimentar $(p=0,004)$. A subescala AE apresentou correlação negativa com a adesão ao consumo de gorduras $(p=0,03)$ e correlação positiva com a DA $(p<0,01)$. Conclusões: $\bigcirc$ tipo de comportamento alimentar mais frequente na amostra foi a restrição cognitiva, que não foi correlacionada com o escore total de adesão. A AE foi associada com maior consumo de gorduras, similar ao encontrado em estudos nacionais e internacionais. Nota-se uma lacuna de estudos que relacionam o comportamento alimentar com a adesão ao tratamento nutricional.

\section{PALAVRAS-CHAVE}

Comportamento alimentar, obesidade, cooperação e adesão ao tratamento, hiperfagia, transtornos do comportamento alimentar.

\section{ABSTRACT}

Objective: To analyze the types of eating behavior in individuals with obesity and correlated with adherence to the adopted treatment. Methods: Cross-sectional study developed at the institution of cardiology in individuals with obesity. The types of eating behavior were analyzed using the scale The Three-Factor Eating Questionnaire - R21 (TFEQ-21) - translated and adapted for Brazilians. Three subscales are addressed: cognitive restriction (CR), emotional eating (EE), and uncontrolled eating (UE). Adherence to nutritional treatment was verified by the instrument developed by the institution, based on the main guidelines for chronic diseases. Results: We analyzed 100 individuals, with a higher prevalence of females (68\%). Regarding adherence, only $25 \%$ have good adherence (score $>60 \%$ ). It was possible to identify the relationship between the BMI and the $C R$, the higher or lower the BMI was the intensity of the $C R(p=0.02)$ There was a positive correlation between $C R$ and adherence to fat $(p=$ 0.02 ) and dietary fiber $(p=0.004)$. The EE subscale showed a negative correlation with adherence to fat consumption ( $p=0.03$ ) and a positive correlation with UE $(p<0.01)$. Conclusions: The most frequent type of eating behavior in the sample was cognitive restriction and was not correlated with the total adherence score. AE was associated with greater consumption of fats, like national and international studies. There is a gap in studies that relate to eating behavior with adherence to nutritional treatment.

\section{KEYWORDS}

Eating behavior, obesity, patient adherence, hyperphagia, eating disorders. 


\section{INTRODUÇÃO}

A obesidade é considerada um problema de saúde pública, com aumento significativo das taxas de sobrepeso e obesidade entre adultos e crianças em diversos países'. No Brasil, em 2018, de acordo com a Vigilância de Fatores de Risco e Proteção para Doenças Crônicas por Inquérito Telefônico (Vigitel), a frequência de excesso de peso foi de $55,7 \%$ e de obesidade, de 19,8\%².

Durante muito tempo, a obesidade foi descrita como uma falha na motivação em perder peso, no entanto atualmente se sabe que ela é uma doença crônica de etiologia multifatorial, na qual se incluem os fatores genéticos, endócrinos, ambientais, sociais, psicológicos e psiquiátricos ${ }^{3}$. Observam-se dificuldades na adesão do tratamento, com crescentes evidências de que em longo prazo o tratamento tradicional da obesidade não é eficaz, pois entre 95\% e 98\% dos pacientes reganham peso após três a quatro anos das intervenções e, além disso, não ocorrem mudanças efetivas no estilo de vida ${ }^{4}$. Na literatura, faltam estudos tenham avaliado a eficácia de programas e os mecanismos que favorecem o sucesso ou a falha no tratamento ${ }^{5}$.

Os indivíduos com obesidade que buscam tratamento especializado para perda de peso podem apresentar maior prevalência de sintomas de ansiedade, depressão, traços de impulsividade e transtornos do comportamento alimentar ${ }^{6}$. Vários padrões de comportamentos alimentares foram identificados e eles incluem o descontrole alimentar (DA), a alimentação emocional (AE) e a restrição cognitiva (RC). O The Three-Factor Eating Questionnaire (TFEQ) é uma escala autoavaliativa amplamente utilizada em indivíduos com sobrepeso/obesidade e eutróficos ${ }^{7}$. Ela foi projetada para avaliar os três fatores da alimentação (DA, AE e RC) e, dessa forma, facilitar a compreensão multidimensional da obesidade que será necessária para delinear o rumo do tratamento. Existem variedades da TFEQ, mas todas avaliam o mesmo fenômeno - a capacidade de restringir os alimentos, o poder da desinibição e o efeito das emoções e a sua relação com o comer.

A RC consiste na adoção de uma posição mental em relação à alimentação com a finalidade de reduzir a ingestão calórica. O indivíduo se impõe obrigações e regras alimentares para manter ou promover a perda de peso ${ }^{8}$. A AE é caracterizada pelo impulso de comer em resposta a emoções, como uma forma de lidar ou fugir de uma emoção. As alterações no humor são gatilhos para a desinibição do controle alimentar e sabe-se que esse comportamento está positivamente associado com o ganho de peso, ansiedade e sintomatologia de depressão 9 . Convencionou-se caracterizar o DA como a perda de autocontrole e consumo excessivo de alimentos, com ou sem a presença de fome. Entre as pessoas com obesidade que buscam tratamento, a incidência de DA varia entre $20 \%$ e $50 \%, 7,10$.
Em revisão de literatura"1, foi avaliada a relação dos padrões de comportamentos alimentares com a obesidade e distúrbios alimentares. Os autores observaram que os padrões de comportamentos alimentares tiveram associação com o índice de massa corporal (IMC), qualidade da dieta, insatisfação corporal e compulsão alimentar. Existe conhecimento limitado sobre a prevalência de padrões de comportamentos alimentares desordenados em pacientes com sobrepeso e obesidade e como os padrões se relacionam com a aderência ao tratamento dietético. Compreender os padrões de comportamentos alimentares é apontado como importante para o manejo da obesidade 5 .

Diante do aumento crescente das taxas de obesidade no país, explorar o comportamento alimentar e relacioná-lo com a obesidade se faz necessário para a construção de uma abordagem nutricional multidimensional que abarca o contexto biológico e psicossocial, que poderá resultar em maior adesão ao tratamento.

O objetivo da pesquisa foi avaliar os tipos de comportamentos alimentares pela escala The Three Factor Eating Questionnaire - R21 (TFEQ-21) em indivíduos com obesidade e correlacionar a adesão ao tratamento proposto em ambulatório de nutrição clínica de uma instituição de cardiologia da cidade de São Paulo.

\section{MÉTODOS}

Trata-se de um estudo observacional transversal com pacientes do ambulatório de nutrição clínica de um instituto de cardiologia localizado na cidade São Paulo. A coleta de dados foi realizada durante agosto de 2018 até agosto 2019. De acordo com o protocolo institucional, todos os pacientes do ambulatório são submetidos ao processo de triagem nutricional que avalia medidas antropométricas, histórico clínico, medicamentos, estilo de vida e consumo alimentar. Dependendo dos critérios (IMC $\geq 30 \mathrm{~kg} / \mathrm{m}^{2}$ e ausência de descompensação de comorbidades), os pacientes são direcionados ao ambulatório de obesidade. Na fase da triagem, é realizada a prescrição dietética hipocalórica, e o paciente retorna ao atendimento para verificar à adesão ao tratamento nutricional proposto na consulta inicial.

Os critérios de inclusão foram indivíduos de ambos os sexos com idade entre 18 e 75 anos, com diagnóstico nutricional de obesidade $\left(\geq 30 \mathrm{~kg} / \mathrm{m}^{2}\right)$ baseado no IMC, com ou sem diagnóstico de diabetes mellitus (DM), hipertensão arterial sistêmica (HAS) e dislipidemia (DLP) e após o segundo retorno ao ambulatório de nutrição. Quanto aos critérios de exclusão, foram pacientes no primeiro atendimento (triagem), gestantes, portadores de transtornos neurocognitivos que impossibilitassem a obtenção de dados fidedignos e usuários de medicamentos para perda de peso, ansiolíticos e 
sedativos. Para o cálculo amostral, o poder do teste utilizado foi de $95 \%$. No total, foram estimados 112 participantes.

Todos os métodos descritos foram submetidos e aprovados pelo Comitê de Ética em Pesquisa do Instituto Dante Pazzanese de Cardiologia, SP, com CAAE: 95590818.8.0000.5462. Todos os participantes foram orientados sobre o Termo de Consentimento Livre e Esclarecido, o anonimato e a possibilidade de desistência a qualquer momento da coleta.

Os tipos de comportamentos alimentares foram avaliados pela escala TFEQ-21 versão traduzida e adaptada para brasileiros com total de 21 questões. Nela são abordadas três subescalas: restrição cognitiva (RC), com itens que aferem a proibição alimentar para influenciar o peso ou a forma corporal; alimentação emocional (AE), com seis itens que mensuram a propensão para comer exageradamente em resposta a estados emocionais negativos, como solidão, ansiedade e depressão; descontrole alimentar (DA), com nove itens que verificam a tendência em perder o controle alimentar na presença ou ausência de fome ${ }^{8}$.

Para determinar os graus de RC, AE e DA, foram utilizadas as instruções propostas pela equipe que desenvolveu o questionário ${ }^{8}$. Para os itens de 1 a 20, foi utilizado o formato de resposta de 4 pontos ( $1=$ totalmente verdade; $2=$ verdade, na maioria das vezes; 3 = falso, na maioria das vezes; 4 = totalmente falso) e uma classificação de 8 pontos para o item 21 ( 1 = comer tudo o que quiser e sempre que quiser e 8 = limitar constantemente a ingestão alimentar, nunca "cedendo"). A média de cada tipo de comportamento foi calculada e transformada em uma escala de 0 a 100 pontos. A pontuação foi categorizada em três níveis: alta (66,67\%-100\%), média (33,34\%-66,66\%) e baixa (0-33,33\%).

A adesão ao tratamento nutricional foi avaliada mediante uma ferramenta desenvolvida na instituição com base teórica nas principais diretrizes brasileiras relacionadas às doenças crônicas não transmissíveis. Contudo, ela está em processo de validação. A ferramenta contém 24 itens subdivididos em nove metas (M): $M 1$ - peso corporal: redução de $\geq 2 \%$ do peso corporal; M2 - circunferência abdominal: redução de $\geq 2 \%$ da circunferência abdominal; M3 - atividade física: um item sobre a prática e frequência de atividade física; M4 - estrutura alimentar: dois itens sobre o fracionamento e consumo de refeições principais e intermediárias; M5 - consumo de açúcar, com quatro itens; M6 - consumo de sódio, com quatro itens; M7 - consumo de gorduras, com quatro itens; M8 - consumo de fibras alimentares, com quatro itens; M9 - consumo de proteínas, com três itens. A média de cada meta é obtida pelo somatório dos valores dos itens que são divididos pelo número de itens, proporcionando pontuação de 0-100\%. O escore total é o resultado da soma de todos as metas, seguido da divisão por 9 . A classificação do escore por metas e total é em boa adesão ( $\geq 60 \%$ ) e baixa adesão $(<60 \%)$.
As análises foram conduzidas com o auxílio do software SPSS versão 26. As variáveis quantitativas foram apresentadas pelo cálculo da média e desvio-padrão e as variáveis qualitativas, pela frequência absoluta e relativa. Para verificar a associação entre os grupos e as variáveis qualitativas, foi utilizado o teste exato de Fisher. As correlações entre as variáveis foram investigadas pelo teste de Spearman. O nível de significância adotado foi de 5\%.

\section{RESULTADOS}

Foram analisados 100 participantes, com idades que variavam de 20 a 75 anos, sendo a idade média e o desvio-padrão, respectivamente, de 58,1 e 11,3. As estatísticas descritivas de sexo, escolaridade, histórico clínico, estilo de vida e adesão ao tratamento nutricional encontram-se na tabela 1. A amostra estudada era majoritariamente feminina (68\%), em comparação com 32\% de participantes do sexo masculino. Com relação à escolaridade, $42 \%$ tinham ensino fundamental, $48 \%$, ensino médio completo e 10\%, ensino superior completo. Nota-se que os participantes estavam no início do tratamento, com a média de três retornos ao ambulatório de nutrição.

Observou-se que 45\% dos indivíduos apresentavam diagnóstico de DM, mas só 3\% utilizavam insulina como parte do tratamento. Em relação a outras doenças crônicas não transmissíveis como HAS, 96\% da amostra apresentava o diagnóstico e 79\%, DLP. Além disso, 62\% dos avaliados relatavam sedentarismo, $82 \%$ declaravam não tabagismo e $18 \%$, cessação. Outro ponto levantado foi o acompanhamento psicológico e histórico de dietas, uma vez que ambos podem afetar o comportamento alimentar. Na amostra, 13\% estavam em acompanhamento psicológico e $71 \%$ já tinham realizado dietas antes do atendimento do ambulatório de nutrição. De acordo com teste exato de Fisher, observamos que o grupo em acompanhamento psicológico representou maior probabilidade de $A E(p=0,01)$.

Nos escores dos tipos de comportamento alimentares da amostra (Tabela 2), destaca-se a média da RC, que foi $76 \%$, seguida do $\mathrm{DA}(30,9 \%)$ e da $\mathrm{AE}(30,1 \%)$. Em relação à classificação, 64\% apresentaram RC alta; enquanto, 15\% tinham AE alta e somente $10 \%$ possuíam DA alta.

Em relação à classificação do estado nutricional de acordo com o IMC, o grau II (35-39,99 kg/m²) foi o mais prevalente, seguido do grau I (30-34,99 kg/m²). Foi possível identificar que quanto maior $\mathrm{I} I \mathrm{MC}$, menor a adesão ao tratamento, com diferença estatística significativa $(p=0,02)$.

De acordo com teste de Spearman, houve correlação significativa entre o IMC e a $\mathrm{RC}(\mathrm{p}=0,02)$; quanto maior $\mathrm{o}$ IMC, menor foi a intensidade da RC (Tabela 2). Nota-se também correlação positiva entre a RC e a meta de adesão de 
gorduras $(p=0,02)$ com o consumo dentro do preconizado, assim como a meta de adesão de fibras alimentares ( $p$ $=0,004)$. A subescala AE apresentou correlação negativa com a meta de adesão de gorduras $(p=0,003)$, ou seja, os indivíduos consumiram valores superiores ao recomendado e uma correlação positiva significativa com o DA $(p<0,01)$. Enquanto o comportamento de DA não apresentou nenhuma associação significativa com as metas de adesão. A RC não se correlacionou com nenhum comportamento alimen$\operatorname{tar}(\mathrm{AE}$ e DA).

Observa-se baixa adesão ao tratamento nutricional, com média de escore total de 50,9\% \pm 17 . Somente $25 \%$ dos participantes tiveram boa adesão ao tratamento nutricional, caracterizada por escore total $\geq 60 \%$ (Tabela 1). Foi identificado na amostra que a meta de atividade física foi a menos atingida, com adesão de $25 \% \pm 37,26$, seguida da meta de peso, que foi de $36 \% \pm 44,28$, e depois da fibra alimentar, que foi de $36,87 \% \pm 27,45$. As metas mais bem-sucedidas foram a de sódio, com média de $78,55 \% \pm 26,26$, seguida da de açúcar, com média de 65,75\% $\pm 28,86$ (Tabela 2).

\section{DISCUSSÃO}

O objetivo do trabalho foi aplicar a escala TFEQ-21 em indivíduos com obesidade e verificar a associação com a adesão ao tratamento nutricional. Houve correlação significativa entre RC e adesão às metas de consumo de gorduras e fibra alimentar. A AE foi correlacionada negativamente com menor adesão à meta de consumo de gorduras (consumindo excessivamente) e positivamente com o DA.

A média do escore da RC foi de 76\%, e $64 \%$ dos participantes apresentavam RC alta, no entanto, refutando a hipótese de que a RC aumentaria a adesão ao tratamento e a redução de peso corporal, no presente estudo esse tipo de comportamento alimentar não foi associado com os escores de boa adesão ao tratamento nutricional e à meta de peso. Sweerts et al. ${ }^{12}$ questionam a viabilidade do constructo da RC pela baixa associação com a ingestão alimentar. Os autores argumentam que as subescalas de restrição não mensuram a ingestão alimentar, mas sim a mediação cognitiva em comer menos do que se deseja.

Johnson et al. ${ }^{13}$ sugerem uma associação entre a redução do peso e o aumento dos escores de RC, entretanto sabe-se que em longo prazo esse mecanismo demonstra-se insustentável e pode estar relacionado ao ganho de peso no futuro. Os altos escores da RC parecem predispor à perda de controle alimentar com episódios de compulsão alimentar, desregulação emocional, redução da autoestima e insatisfação corporal. O reganho de peso após a perda de peso é um fenômeno comum. Melby et al. ${ }^{14}$ descrevem o energy gap como uma pressão biológica responsável pela recuperação

Tabela 1. Estatísticas descritivas de sexo, escolaridade, histórico clínico, estilo de vida e adesão ao tratamento nutricional e sua relação com os tipos de comportamentos alimentares de acordo com teste de exato de Fisher $(n=100)$

\begin{tabular}{|c|c|c|c|c|}
\hline \multirow[b]{2}{*}{ Variáveis } & \multirow[b]{2}{*}{ n (\%) } & \multicolumn{3}{|c|}{ Tipos de comportamentos alimentares } \\
\hline & & $\begin{array}{c}\text { RC } \\
\text { (p-valor) }\end{array}$ & $\begin{array}{c}\mathrm{AE} \\
\text { (p-valor) }\end{array}$ & $\begin{array}{c}\text { DA } \\
\text { (p-valor) }\end{array}$ \\
\hline Sexo & & 0,23 & 0,89 & 0,76 \\
\hline Feminino & $68(68 \%)$ & & & \\
\hline Masculino & $32(32 \%)$ & & & \\
\hline Escolaridade & & 0,71 & 0,92 & 0,32 \\
\hline Ensino fundamental & $42(42 \%)$ & & & \\
\hline Ensino médio & $48(48 \%)$ & & & \\
\hline Ensino superior & $10(10 \%)$ & & & \\
\hline Diabetes mellitus & $45(45 \%)$ & 0,71 & 0,75 & 0,37 \\
\hline Hipertensão arterial sistêmica & $96(96 \%)$ & 0,10 & 0,37 & 0,55 \\
\hline Dislipidemia & $79(79 \%)$ & 0,10 & 0,20 & 0,93 \\
\hline Sedentarismo & $62(62 \%)$ & 0,81 & 0,28 & 0,85 \\
\hline Tabagismo & & 0,16 & 0,08 & 0,51 \\
\hline Não tabagista & $82(82 \%)$ & & & \\
\hline Ex-tabagista & $18(18 \%)$ & & & \\
\hline Acompanhamento psicológico & $13(13 \%)$ & 0,93 & $0,01^{*}$ & 0,49 \\
\hline Histórico de dietas & $71(71 \%)$ & 0,74 & 0,31 & 0,94 \\
\hline Boa adesão ao tratamento** & $25(25 \%)$ & 0,89 & 0,83 & 0,88 \\
\hline 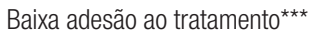 & 75 (75\%) & 0,90 & 0,79 & 0,91 \\
\hline
\end{tabular}

n: número da amostra; RC: restrição cognitiva; AE: alimentação emocional; DA: descontrole alimentar. ${ }^{*} p<0,05 .{ }^{* \star}$ Escore total $\geq 60 \%$. ${ }^{\star \star \star}$ Escore total $<60 \%$. 
Tabela 2. Estatística descritiva do índice de massa corporal, circunferência abdominal, \% de tipos de comportamentos alimentares e escore de adesão ao tratamento nutricional e a correlação com os tipos de comportamentos alimentares de acordo com o teste de Spearman $(n=100)$

\begin{tabular}{|c|c|c|c|c|}
\hline \multirow{2}{*}{ Variáveis } & \multirow{2}{*}{ Média \pm desvio-padrão } & \multicolumn{3}{|c|}{ Tipos de comportamentos alimentares } \\
\hline & & $\begin{array}{c}\mathrm{RC} \\
\text { (p-valor) }\end{array}$ & $\underset{\text { (p-valor) }}{\mathrm{AE}}$ & $\underset{\text { (p-valor) }}{\mathrm{DA}}$ \\
\hline Índice de massa corporal (kg/m²) & $39,4 \pm 5,8$ & $-0,02^{*}$ & 0,72 & 0,88 \\
\hline Circunferência abdominal (cm) & $116,5 \pm 12,2$ & 0,95 & 0,36 & 0,27 \\
\hline Restrição cognitiva (\%) & $76,0 \pm 21,3$ & - & 0,13 & 0,89 \\
\hline Alimentação emocional (\%) & $30,1 \pm 32,7$ & 0,13 & - & $0,00^{*}$ \\
\hline Descontrole alimentar (\%) & $30,9 \pm 25,9$ & 0,89 & $0,00^{*}$ & - \\
\hline Meta de peso (\%) & $36,0 \pm 44,2$ & 0,91 & 0,76 & 0,21 \\
\hline Meta de circunferência abdominal (\%) & $42,0 \pm 48,5$ & 0,99 & 0,85 & 0,91 \\
\hline Meta de atividade física (\%) & $25,0 \pm 37,2$ & 0,20 & 0,51 & 0,25 \\
\hline Meta de dieta (\%) & $58,4 \pm 24,9$ & 0,20 & 0,45 & 0,12 \\
\hline Meta de açúcar (\%) & $65,7 \pm 28,8$ & 0,26 & 0,21 & 0,51 \\
\hline Meta de sódio (\%) & $78,5 \pm 26,2$ & 0,32 & 0,21 & 0,58 \\
\hline Meta de gordura (\%) & $60,7 \pm 29,9$ & $0,02^{*}$ & $-0,03^{*}$ & 0,64 \\
\hline Meta de fibra alimentar (\%) & $36,8 \pm 27,4$ & $0,04^{*}$ & 0,15 & 0,54 \\
\hline Meta de proteína (\%) & $57,5 \pm 22,0$ & 0,13 & 0,20 & 0,57 \\
\hline Escore total (\%) & $50,9 \pm 17,0$ & 0,06 & 0,30 & 0,48 \\
\hline
\end{tabular}

n: número da amostra; RC: restrição cognitiva; AE: alimentação emocional; DA: descontrole alimentar. * $p<0,05$.

do peso perdido, aumentando a fome e reduzindo o gasto energético. Diversos fatores como circulação de leptina, grelina, insulina, atividade hipotalâmica e expressão de neuropeptídios contribuem para o energy gap induzido por dietas para redução de peso.

Os impactos da RC no peso e IMC são controversos, com correlações negativas ou positivas. No presente estudo, houve associação negativa entre o IMC e a RC $(p=0,02)$, na qual, quanto maior o IMC, menor era a RC. Em ensaio clínico de Nurkkala et al. ${ }^{15}$, a alta pontuação em RC foi relacionada com maior IMC, insucesso na manutenção do peso e comportamentos alimentares disfuncionais. No estudo, $46 \%$ dos indivíduos do grupo intervenção e $24 \%$ do grupo controle conseguiram manter a perda de $5 \%$ do peso até o final do ensaio clínico. Em follow-up de três anos, a RC aumentou em até 16 pontos no grupo intervenção e 7 pontos no grupo controle $(p=0,004)$, e o DA não diferiu entre os grupos. A AE foi maior entre o grupo controle $(p=0,003)$, no entanto foi decrescendo ao longo do tempo no grupo controle quando comparado com o grupo intervenção $(p=0,0015)$.

Sweerts et al..$^{12}$ utilizaram a escala TFEQ-21 em 507 franceses e identificaram associação positiva significativa entre o RC e o IMC $(p=0,01)$ e o peso $(p=0,08)$, observando que quanto maior o peso corporal, maiores os escores da RC. Em estudo de Chacko et al. ${ }^{16}$, participantes com obesidade severa (IMC $\geq 45 \mathrm{~kg} / \mathrm{m}^{2}$ ) apresentaram correlação significativa com comportamentos alimentares disfuncionais, como
DA e AE. Estudos de imagem cerebral indicam a associação entre peso e restrição alimentar, demonstrando que o IMC elevado foi relacionado com a atividade metabólica reduzida em áreas cerebrais ligadas ao controle executivo (pré-frontal e dorsolateral) ${ }^{12}$. Os indivíduos que mantêm a perda significativa de peso aumentam a atividade neural em áreas de controle como córtex pré-frontal dorsal, estriado e lobo cerebelar anterior ${ }^{13}$.

No presente estudo, não foi encontrada correlação significativa entre AE e o consumo de açúcares. Os resultados foram semelhantes com o estudo de Strengell et al. ${ }^{17}$, em que a $\mathrm{AE}$ não foi correlacionada com a ingestão de sacarose, lipídeos, proteínas, fibras e álcool. No entanto, notou-se, na amostra do presente estudo, a correlação positiva entre a AE e o DA, corroborando a pesquisa de Natacci e Ferreira Júnior ${ }^{8}$, que também identificaram associações entre os comportamentos. Cappelleri et al.? encontraram a AE positivamente associada com o DA, mas também com a RC, enquanto Chacko et al. ${ }^{16}$ observaram, em indivíduos com obesidade, que altos escores de AE foram correlacionados fortemente com o DA $(r=0,42)$ e a $R C$ foi menos correlacionada com $A E$ $(r=0,10)$ e $D A(r=0,06)$.

Lopez-Cepero et al. ${ }^{18}$ observaram que os altos escores de AE foram associados com hipertensão arterial $(p<0,01)$ e DM ( $p<0,01)$, diferentemente do presente estudo, em que a $A E$ não foi correlacionada com o histórico clínico. No entanto, a AE foi correlacionada negativamente com a meta 
de consumo de gorduras $(p=0,03)$, demonstrando possível ingestão exagerada de alimentos fontes de gorduras.

No presente estudo, o comportamento de DA não apresentou correlação significativa com a adesão e a RC, diferente do estudo de Nurkkala et al. ${ }^{15}$, em que a RC demonstrou elevação do DA entre indivíduos com excesso de peso. Altos níveis de DA são associados com uma preferência por alimentos salgados e gordurosos ${ }^{18}$, mas no presente estudo não foi encontrada associação significativa com as metas de gorduras e sódio.

Chackon et al. ${ }^{16}$ observaram associação positiva entre altos escores de AE e DA e dificuldades na redução no tamanho de porções, ingestão calórica, de carboidratos e gorduras. A mudança de comportamento deve visar à redução da AE e DA, desenvolvendo métodos que auxiliem os indivíduos a reconhecerem as situações de riscos, principalmente os indivíduos com baixa adesão e muitas recaídas durante o processo de mudança de estilo de vida ${ }^{17}$. Agora, Keränen et al. ${ }^{19}$ relataram que o aumento da $\mathrm{RC}$ com a redução da DA e AE foi associado com a manutenção da perda de peso em longo prazo, no entanto ressaltaram que existem dois tipos de RC rígida e flexível. A flexível é mais favorável para mudanças, visto que que a rigidez pode aumentar a desinibição alimentar.

Outro ponto analisado no estudo foi a adesão ao tratamento nutricional, destacando-se que $75 \%$ dos participantes apresentavam baixa adesão ao tratamento nutricional (média do escore total $<60 \%$ ). As principais barreiras na prevenção e tratamento da obesidade são a adesão, a manutenção da perda de peso em longo prazo e o estigma do peso ${ }^{20,21}$. Em programas tradicionais para controle e prevenção da obesidade, o foco é exclusivamente no manejo de indicadores como peso e IMC. Varela et al..$^{20}$ sugerem a mudança dos tratamentos com o foco na compreensão do sujeito que vai receber a intervenção, e não só no conhecimento sobre os mecanismos fisiopatológicos da obesidade. Recomendase o investimento em estratégias voltadas à promoção mais ampla da saúde com mudanças de comportamentos, abarcando as influências exercidas por fatores ambientais, preocupações com a imagem corporal, estigmatização do peso e prevenção de transtornos alimentares.

Acompanhando a proposta, recentemente surgiram estudos que investigam aspectos da autorregulação do comportamento alimentar. O intuitive eating (comer intuitivo) é uma abordagem promissora, caracterizada como um processo dinâmico integrativo que sintoniza mente, corpo e comida, regulando a ingestão pelos sinais internos de fome e saciedade ${ }^{22}$. Hazzard et al.22, em estudo longitudinal, observaram que os participantes com altos escores na escala de comer intuitivo tiveram menores riscos para sintomas depressivos, baixa autoestima, insatisfação corporal e DA ( $p<$ 0,01) em follow-up de oito anos.
Nesse contexto, a abordagem como o Health at Every Size ${ }^{\circ}\left(\mathrm{HAES}^{\circ}\right)$ também é apontada como uma alternativa para a promoção de comportamentos alimentares saudáveis e a prática de atividades físicas prazerosas e sustentáveis, para pessoas de diferentes tamanhos. Ulian et al. ${ }^{23}$, em ensaio clínico randomizado com grupo controle, encontraram que mulheres com obesidade do grupo intervenção intensiva baseada no HAES tiveram redução no consumo diário de alimentos ultraprocessados ( $p<0,0001)$, aumento no consumo diário de frutas $(p=0,003)$ e vegetais $(p=0,012)$ e melhora nas atitudes corporais $(p<0,05)$ e na qualidade de vida $(p=0,05)$, enquanto as medidas antropométricas não apresentaram mudanças significativas.

O mindfulness (atenção plena) também é relatado como uma abordagem capaz de promover a autorregulação. Nas intervenções baseadas em mindfulness, a perda de peso não é o desfecho principal, e sim outros desfechos como redução do DA e AE ${ }^{24}$, mas Daubenmier et al..$^{24}$ avaliaram as mudanças de peso do grupo intervenção baseada em mindfulness e o grupo de espera (controle). Os autores observaram que o grupo intervenção manteve o peso, enquanto o grupo controle ganhou em média 1,7 kg em follow-up de quatro meses. Fuentes Artiles et al. ${ }^{25}$ conduziram revisão sistemática com metanálise para avaliar a eficácia das intervenções baseadas em mindful eating (comer com atenção plena) e intuitive eating no controle de peso. Os autores constataram que os grupos de intervenção apresentaram perda de peso mais significativa quando comparados com o grupo controle sem dieta $(p=0,005)$.

O presente estudo apresenta algumas limitações. Sabe-se que o fato de o participante já ter recebido uma prescrição dietética na primeira consulta pode estimular a restrição alimentar, portanto esperava-se que a RC fosse mais prevalente na população estudada. Outra limitação foi utilizar uma ferramenta para mensurar a adesão ao tratamento nutricional que ainda está em processo de validação. O estudo também utilizou um questionário de autorrelato com um potencial viés de falsa resposta, visto que a escala trata de questões que muitas vezes são encaradas como "embaraçosas" e as respostas podem não ser condizentes com a realidade. Dado que muitos participantes poderiam apresentar dificuldades na leitura e compreensão das questões, optou-se pela aplicação pelo próprio pesquisador, o que é capaz de intimidar o participante.

O número de participantes foi inferior ao cálculo amostral, possivelmente gerando pouca representatividade amostral. Além disso, a forma de seleção foi realizada de modo conveniente em um ambulatório de nutrição com foco na cardiologia, portanto as conclusões não podem ser extrapoladas para outras populações. Outra limitação foi a ausência de comparação entre grupos, de acordo com adesão e tipos de comportamentos alimentares mais evidentes. Também não houve o controle de fatores de confusão como sexo, idade, escolaridade, etnia e condição sociodemográfica. 
Com o delineamento transversal da pesquisa, não foi possível estabelecer uma relação de causalidade entre os comportamentos alimentares e adesão ao tratamento nutricional. Além disso, os participantes estavam no início do tratamento, evidenciado pela média de três retornos, e os comportamentos de AE e DA não se manifestaram com intensidade, mas é possível especular que poderiam desenvolvê-los, dado que o escore médio da RC foi classificado como alto.

Ressalta-se que o presente estudo teve a proposta de fazer uma avaliação diagnóstica, mas recomenda-se também o delineamento prospectivo para avaliar a função prognóstica da escala, ou seja, avaliar em longo prazo o impacto dos comportamentos alimentares na adesão ao tratamento nutricional.

\section{CONCLUSÕES}

Há uma lacuna na literatura científica brasileira sobre a relação entre os comportamentos alimentares e a adesão ao tratamento nutricional em indivíduos com obesidade. O presente estudo contribuiu para preencher parte dessa lacuna, identificando que o tipo de comportamento alimentar mais prevalente na amostra foi a $\mathrm{RC}$, que se correlacionou com a adesão às metas de consumo de fibra alimentar e gordura, mas não foi associada com os demais comportamentos alimentares (AE e DA), diferentemente do que é descrito na literatura. A AE e o DA foram correlacionados positivamente entre si, e a AE foi associada negativamente com menor adesão às metas de gorduras, similar aos estudos nacionais e internacionais.

Diante dos resultados, é importante aplicar escalas que avaliam os tipos de comportamentos alimentares na triagem nutricional e expandir a visão que se tem do indivíduo, realizando condutas mais condizentes, reduzindo comportamentos alimentares disfuncionais e aumentando a adesão ao tratamento nutricional.

\section{CONTRIBUIÇÕES INDIVIDUAIS}

Leonardo Domingos Biagio - Responsável pela concepção, desenho do estudo, coleta de dados, análise e interpretação dos dados e elaboração do artigo.

Priscila Moreira - Auxílio na aplicação das escalas, orientações específicas sobre o público atendido, análise e interpretação dos dados.

Cristiane Kovacs Amaral - Revisão crítica do conteúdo e aprovação da versão final.

\section{CONFLITO DE INTERESSES}

Todos autores declaram ausência de conflito de interesses.

\section{AGRADECIMENTOS}

Agradecimento especial à Cristiane Kovacs, chefe do Ambulatório de Nutrição Clínica do Instituto Dante Pazzanese de Cardiologia e coordenadora da área de Nutrição da Residência, que acreditou na pesquisa de um tema ainda não explorado na instituição. Somos gratos pela confiança e parceria.

\section{REFERÊNCIAS}

1. Mastellos N, Gunn LH, Felix LM, Car J, Majeed A. Transtheoretical model stages of change for dietary and physical exercise modification in weight loss management for overweight and obese adults. Cochrane Database Syst Rev. 2014;(2):CD008066.

2. Brasil. Ministério da Saúde. Secretaria da Vigilância em Saúde. Departamento de Análise em Saúde e Vigilância de Doenças não Transmissíveis. Vigitel Brasil 2018: vigilância de fatores de risco e proteção para doenças crônicas por inquérito telefônico: estimativas sobre frequência e distribuição sociodemográfica de fatores de risco e proteção para doenças nas capitais de 26 estados. Brasília: Ministério da Saúde; 2019. Disponível em: https://portalarquivos2.saude.gov.br/images/pdf/2019/julho/25/vigitel-brasil-2018.pdf. Acesso em: 16 abr. 2020

3. Macpherson-Sánchez AE. Integrating fundamental concepts of obesity and eating disorders: Implications for the obesity epidemic. Am J Public Health. 2015;105(4):71-85.

4. Cadena-Schlam L, López-Guimerà G. Ingesta intuitiva: Un nuevo abordaje del comportamiento alimentario. Nutr Hosp. 2015;31(3):995-1002.

5. Teixeira PJ, Carraça EV, Marques MM, Rutter H, Oppert JM, De Bourdeaudhuij I, et al. Successful behavior change in obesity interventions in adults: A systematic review of selfregulation mediators. BMC Med. 2015;13(1):1-16.

6. Pisciolaro F, Azevedo AP. Transtornos alimentares e obesidade. In: Alvarenga M, Scagliusi FB, Philippi ST. Nutrição e Transtornos Alimentares: avaliação e tratamento. Barueri, SP: Manole; 2011. p. 87-98.

7. Cappelleri JC, Bushmakin AG, Gerber RA, Leidy NK, Sexton CC, Lowe MR, et al. Psychometric analysis of the Three-Factor Eating Questionnaire-R21: Results from a large diverse sample of obese and non-obese participants. Int J Obes. 2009;33(6):611-20.

8. Natacci LC, Ferreira Júnior M. The Three Factor Eating Questionnaire - R21: Tradução para o português e aplicação em mulheres brasileiras. Rev Nutr. 2011;24(3):383-94.

9. Wehling H, Lusher JM. Cognitive and Emotional Influences on Eating Behaviour: A Qualitative Perspective. Nutr Metab Insights. 2019;12(11):786-38.

10. Bohrer BK, Forbush KT, Hunt TK. Are common measures of dietary restraint and disinhibited eating reliable and valid in obese persons? Appetite. 2015;87(1):344-51.

11. Bryant EJ, Rehman J, Pepper LB, Walters ER. Obesity and Eating Disturbance: the Role of TFEQ Restraint and Disinhibition. Curr Obes Rep. 2019;8(4):363-72.

12. Sweerts SJ, Fouques D, Lignier B, Apfeldorfer G, Lucia KK. Relation between cognitive restraint and weight: Does a content validity problem lead to a wrong axis of care? Clin Obes. 2019;9(5):1-5.

13. Johnson F, Pratt M, Wardle J. Dietary restraint and self-regulation in eating behavior. Int J Obes. 2012;36(5):665-74.

14. Melby CL, Paris HL, Foright RM, Peth J. Attenuating the biologic drive for weight regain following weight loss: Must what goes down always go back up? Nutrients. 2017;9(5):121.

15. Nurkkala M, Kaikkonen K, Vanhala ML, Karhunen L, Keränen A, Korpelainen R. Eating Behaviors Lifestyle intervention has a beneficial effect on eating behavior and long-term weight loss in obese adults. Eat Behav. 2015;18(1):179-85

16. Chacko SA, Chiodi SN, Wee CC. Recognizing disordered eating in primary care patients with obesity. Prev Med. 2015;72(1):89-94.

17. Strengell K, Savolainen MJ, Laitinen JH, Kera A. Effect of weight loss intervention on the association between eating behavior measured by TFEQ-18 and dietary intake in adults. Appetite. 2011;56(1):156-62. 
18. Lopez-Cepero A, Frisard CF, Lemon SC, Rosal MC. Association of Dysfunctional Eating Patterns and Metabolic Risk Factors for Cardiovascular Disease among Latinos. I Acad Nutr Diet. 2018;118(5):849-56.

19. Keränen AM, Savolainen MJ, Reponen AH, Kujari ML, Lindeman SM, Bloigu RS, et al. The effect of eating behavior on weight loss and maintenance during a lifestyle intervention. Prev Med. 2009;49(1):32-8.

20. Varela C, Andrés A, Saldaña C. The behavioral pathway model to overweight and obesity: coping strategies, eating behaviors and body mass index. Eat Weight Disord. 2019;(1):23456.

21. Tylka TL, Annunziato RA, Burgard D, Daníelsdóttir S, Shuman E, Davis C, et al. The WeightInclusive versus Weight-Normative Approach to Health: Evaluating the Evidence for Prioritizing Well-Being over Weight Loss. J Obes. 2014:983495.
22. Hazzard VM, Telke SE, Simone M, Anderson LM, Larson NI, Neumark-Sztainer D. Intuitive eating longitudinally predicts better psychological health and lower use of disordered eating behaviors: findings from EAT 2010-2018. Eat Weight Disord. 2020.

23. Ulian MD, Sato PDM, Fabiana B, Je A, Campos-Ferraz PL De, Coelho D, et al. Effects of a new intervention based on the Health at Every Size approach for the management of obesity: The "Health and Wellness in Obesity" study. Plos One. 2018;13(7):1-19.

24. Daubenmier J, Kristeller J, Hecht FM, Maninger N, Kuwata M, Jhaveri K, et al. Mindfulness intervention for stress eating to reduce cortisol and abdominal fat among overweight and obese women: An exploratory randomized controlled study. J Obes. 2011;2011:651936.

25. Fuentes Artiles R, Staub K, Aldakak L, Eppenberger P, Rühli F, Bender N. Mindful eating and common diet programs lower body weight similarly: Systematic review and metaanalysis. Obes Rev. 2019;20(11):1619-27. 


\section{Comportamento alimentar em obesos e sua} correlação com o tratamento nutricional

Eating behavior in obesity and its correlation with nutritional treatment

DOI: $10.1590 / 0047-2085000000280$

Where you read:

Received in: May/10/2020. Approved in: May/20/2020

Should read:

Received in: Mar/02/2020. Approved in: May/10/2020

DOI: $10.1590 / 0047-2085000000320$ 